\title{
THE METHOD OF FUNDEMENTAL SOLUTIONS FOR THE MULTI- DIMENSIONAL WAVE EQUATIONS
}

\author{
Meng-Huang Gu \\ Department of Civil Engineering and Hydrotech Research Institute, National Taiwan University, Taipei 10617, Taiwan. \\ R.O.C. \\ Chia-Ming Fan \\ Department of Harbor and River Engineering \& Computation and Simulation Center, National Taiwan Ocean University, \\ Keelung 20224, Taiwan, R.O.C. \\ Der-Liang Young \\ Department of Civil Engineering and Hydrotech Research Institute, National Taiwan University, Taipei 10617, Taiwan. \\ R.O.C., dlyoung@ntu.edu.tw
}

Follow this and additional works at: https://jmstt.ntou.edu.tw/journal

Part of the Engineering Commons

\section{Recommended Citation}

Gu, Meng-Huang; Fan, Chia-Ming; and Young, Der-Liang (2011) "THE METHOD OF FUNDEMENTAL SOLUTIONS FOR THE MULTI-DIMENSIONAL WAVE EQUATIONS," Journal of Marine Science and Technology. Vol. 19: Iss. 6, Article 2. DOI: $10.51400 / 2709-6998.2200$

Available at: https://jmstt.ntou.edu.tw/journal/vol19/iss6/2

This Research Article is brought to you for free and open access by Journal of Marine Science and Technology. It has been accepted for inclusion in Journal of Marine Science and Technology by an authorized editor of Journal of Marine Science and Technology. 
THE METHOD OF FUNDEMENTAL SOLUTIONS FOR THE MULTI-DIMENSIONAL WAVE EQUATIONS

\section{Acknowledgements}

The National Science Council (NSC) of Taiwan is gratefully acknowledged for providing the financial support to carry out the present work under the grant number: NSC-97-2221-E-002-247-MY3 and NSC-96-2221-E-002-127-MY3. We also deeply appreciated about some importance suggestions by Professor C.S. Chen in Department of Mathematics University of Southern Mississippi, USA. 


\title{
THE METHOD OF FUNDEMENTAL SOLUTIONS FOR THE MULTI-DIMENSIONAL WAVE EQUATIONS
}

\author{
Meng-Huang Gu*, Chia-Ming Fan**, and Der-Liang Young*
}

Key words: method of fundamental solutions, method of particular solutions, Houbolt method, wave equation.

\begin{abstract}
In this paper, a meshless method is developed for solving multi-dimensional wave equations. The proposed method is based on the method of particular solution (MPS), the method of fundamental solutions (MFS) and the Houbolt finite difference (FD) method. The wave equation is considered as a Poisson-type equation with the time-dependent source term. The Houbolt method is applied to avoid the difficult problems for dealing with the initial conditions in forming the linear algebra system. The works of space discretization are dependent on the method of particular solution and the method of fundamental solutions. There are three numerical examples considered in this paper, such as the string vibration and wave vibration problems. Numerical validations have proven that the proposed method is a highly efficient and accurate meshless numerical tool for solving wave equations in engineering and sciences by comparing with analytical solution and other numerical solutions.
\end{abstract}

\section{INTRODUCTION}

Although there are many numerical methods available for solving hyperbolic-type partial differential equations, the development of highly accurate and efficient wave solvers remains an important and challenging work in computational physics. The wave equations govern many physical problems such as water wave propagation in water bodies, the stress wave in an elastic solid and sound wave propagation in a medium, etc. In this paper, a meshless numerical method based on the method of particular solutions (MPS), the method of the

Paper submitted 02/25/10; revised 05/20/10; accepted 06/22/10. Author for correspondence: Der-Liang Young (e-mail: dlyoung@ntu.edu.tw).

*Department of Civil Engineering and Hydrotech Research Institute, National Taiwan University, Taipei 10617, Taiwan. R.O.C.

**Department of Harbor and River Engineering \& Computation and Simulation Center, National Taiwan Ocean University, Keelung 20224, Taiwan, R.O.C. fundamental solutions (MFS) and the Houbolt finite difference (FD) scheme is proposed for dealing with multi-dimensional wave problems.

Generally speaking, the so-called meshless numerical schemes can be roughly classified into domain-type and boundary-type methods. The domain-type meshless numerical methods such as the smoothed particle hydrodynamics (SPH) [15] and the multi-quadric (MQ) collocation method [9], etc. were well developed for solving partial differential equations. The boundary-type meshless methods such as the MFS [7, 31], the hyper-singular meshless method (HMM) [27] and the Trefftz method $[5,6,8]$, etc. have also been developed to obtain solutions of homogeneous partial differential equations. In this study, we propose an extended scheme of the MFS to solve hyperbolic-type partial differential equations such as wave equations.

The MFS was firstly proposed for approximating the solutions of the elliptic-type partial differential equation [17, 18]. Furthermore, the two-stage MPS-MFS was proposed to solve Poisson's equations [11]. In above studies, the MFS captured many focuses of researcher in solving elliptic-type partial differential equations. For time-dependent problems, the MFS was applied to solve the homogeneous or inhomogeneous problems either by the time-marching MFS $[2,22]$, the unified time-space MFS with diffusion fundamental solution [32] or the so-called eigenfunction expansion MFS [26]. These studies also focused on the solution of parabolic-type partial differential equations.

Although the MFS can successfully deal with the elliptic or parabolic problems, it is not so easy to directly handle hyperbolic-type problems such as wave or advection phenomena. In reference [29], the Eulerian-Lagrangian method (ELM) was combined with the MFS to deal with the multi-dimensional advection-diffusion problems (called Eulerian-Lagrangian method of fundamental solutions, ELMFS). The ELMFS has been applied effectively to solve the nonlinear Burgers' equations [28], the pure advection equations and one-dimensional wave equation $[12,13]$, etc.

Although the MFS is a powerful tool for solving partial differential equations, it is still difficult to directly solve wave equation by the MFS. Because the fundamental solution of wave equation always accompanies the Dirac delta function 
(or Heaviside step function). When the fundamental solutions of the wave equation are used for the implementation of the MFS, we have to face the difficult problems such as differentiating the Dirac delta function (or Heaviside step function) with respect to the time domain for building the linear algebra system. This will induce difficult singularity problems for computer calculation by the MFS. Another well-known process for analyzing the wave equation is to transform the physical variables from time domain into the frequency domain [16, 19]. According to above approach, the initial and boundary value problems become the pure boundary value problems, but sometimes it is more difficult to directly capture the transient phenomena of the wave field via this mode decomposition approach.

The D'Alembert formulation [4, 24] is considered as another effective way for avoiding the problems of the Dirac delta function (or Heaviside step function) in time-space domains. The D'Alembert formula was combined with the decomposition method to obtain the solution of the wave equation in infinite domain [24]. The ELMFS was combined with the D'Alembert formula for directly solving the onedimensional wave equation $[12,13]$. The D'Alembert formula can reduce the one-dimensional wave equation to two advection equations with opposite direction wave propagation speed. The ELMFS then approximates the solutions of the advection equation system. Although the D'Alembert formula can handle the time-space Cauchy problems, the reductions also cause problems in treating the boundary conditions.

The proposed coupled MPS-MFS is a kind of meshless method which includes the characteristics of both domain- and boundary-type meshless methods. The proposed MPS-MFS model transforms the wave equation to the Poisson-type equation with a time-dependent source term. Thus the hyperbolic problem becomes an elliptic boundary value problem. The dual reciprocity boundary element method (DRBEM) is similar to the coupled MPS-MFS model, which was developed for solving the wave equation [21]. However, the DRBEM requires the time-consuming construction of good surface mesh and accurate numerical quadrature. The coupled MPS-MFS meshless schemes [1] were developed to solve non-homogeneous elliptic problems. Besides, the schemes of coupled MPS-MFS were applied to analyze the conductive problems for functionally graded materials [23] and time-dependent partial differential equations [10].

In this paper, the time-dependent loading of the system equations is handled by the Houbolt method [14, 20, 25, 30]. Then the physical solution is separated into the particular and the homogeneous solutions at each time step. The particular solution is dependent on radial basis functions (RBFs) while the homogeneous solution is dealt with by the Laplace fundamental solution. In the following sections, we will explain the details of the time-marching MPS-MFS wave model and the numerical procedures. Three numerical examples are provided for validating the proposed meshless method. All of the numerical results compare well with the analytical solu- tions or solutions obtained by finite difference method (FDM) or the finite element method (FEM). From the numerical tests, it is evident that the proposed transient MPS-MFS wave model is a promising meshless numerical tool for physical applications.

\section{GOVERNING EQUATION}

The multi-dimensional wave equation can be written as:

$$
\frac{\partial^{2} \phi}{\partial t^{2}}=c^{2} \nabla^{2} \phi, \text { in } \Omega, t>0,
$$

where, $\Omega$ is the computational domain with boundary $\partial \Omega=$ $\partial \Omega_{1} \cup \partial \Omega_{2}, \phi(\vec{x}, t)$ is the physical variable, $c$ is the wave speed, $t$ denotes time and $\vec{x}$ is the space vector. In Cauchy or initial value problems, the initial conditions are described as follows:

$$
\begin{gathered}
\left.\phi(\vec{x}, t)\right|_{t=0}=I_{I}(\vec{x}), \\
\left.\phi(\vec{x}, t)\right|_{t=0}=I_{I}(\vec{x}),
\end{gathered}
$$

where, the subscripts $I$ and $I I$ are used to denote first- and second-kind initial conditions, respectively. The boundary conditions are listed as follows:

$$
\begin{gathered}
\left.\phi(\vec{x}, t)\right|_{\vec{x} \in \partial \Omega_{1}}=a_{D}(\vec{x}, t), \\
\left.\frac{\partial \phi(\vec{x}, t)}{\partial n}\right|_{\vec{x} \in \partial \Omega_{2}}=a_{N}(\vec{x}, t),
\end{gathered}
$$

where, $\vec{n}$ is the unit normal vector outward to the boundary, $a$ is a function of space and time. The subscripts $D$ and $N$ denote Dirichlet- and Neumann-type boundary conditions, respectively.

\section{NUMERICAL METHOD}

The governing equation is considered as a Poisson-type equation with time-dependent source term. The governing equation and boundary condition can be rewritten as follows:

$$
\begin{gathered}
\nabla^{2} \phi=\frac{1}{c^{2}} \frac{\partial^{2} \phi}{\partial t^{2}}, \text { in } \Omega, t>0, \\
B(\phi)=b(\vec{x}, t), \vec{x} \in \partial \Omega, t>0 .
\end{gathered}
$$

where, $B()$ denotes the boundary operator. In order to deal with the transient term of wave equation, the Houbolt method is selected to discrete the time operators. The Houbolt method $[14,20,25,30]$ is an three-steps implicit and unconditionally 
stable time-integration scheme which can be obtained by the Lagrange interpolation of the wave potential $\phi$ from time level $t^{n-2}=(n-2) \Delta t$ through to time level $t^{n+1}=(n+1) \Delta t$. The time operators as the velocity and acceleration of wave field are approximated as follows:

$$
\begin{gathered}
\left(\frac{\partial \phi}{\partial t}\right)^{n+1} \approx \frac{1}{6 \Delta t}\left(11 \phi^{n+1}-18 \phi^{n}+9 \phi^{n-1}-2 \phi^{n-2}\right), \\
\left(\frac{\partial^{2} \phi}{\partial t^{2}}\right)^{n+1} \approx \frac{1}{\Delta t^{2}}\left(2 \phi^{n+1}-5 \phi^{n}+4 \phi^{n-1}-\phi^{n-2}\right) .
\end{gathered}
$$

Here, $\Delta t$ is the time-interval and the superscripts of $\phi$ represent the time level $\phi^{n}(\vec{x})=\phi\left(\vec{x}, t^{n}\right)$. After dealing with the time domain problems by using the Houbolt FD scheme, we obtain a Poisson-type equation as the following.

$$
\left(\nabla^{2} \phi\right)^{n+1}=\frac{1}{c^{2} \Delta t^{2}}\left(2 \phi^{n+1}-5 \phi^{n}+4 \phi^{n-1}-\phi^{n-2}\right)
$$

In the MPS-MFS, the solution of the problem can be written as:

$$
\phi^{n+1}=\phi_{P}^{n+1}+\phi_{H}^{n+1}
$$

where, $\phi_{P}$ is the particular solution which satisfies the nonhomogeneous equation and $\phi_{H}$ is the homogeneous solution which satisfies the Laplace equation. The particular solution $\phi_{P}$ can be approximated by the radial basis functions (RBFs) as the following:

$$
\phi_{P}^{n+1}=\sum_{j=1}^{N_{F}} \beta_{j}^{n+1} F\left(r_{j}\right),
$$

Here, $F()$ is the integrated radial basis function and $\beta_{j}$ is the coefficient of the basis function, $N_{F}$ is the number of the field points and the subscript $j$ denotes the index of the collocation points. A typical distribution of the field and source points is depicted in Fig. 1. The function $F($ ) can be obtained by analytical integration from the following equation:

$$
\nabla^{2} F(r)=f(r)
$$

where, $f(r)$ is the radial basis function. In order to demonstrate the conveniences of RBFs, the local type RBF as compactly supported RBF (CSRBF) and global type RBF as multiquadric RBF (MQRBF) are selected for describing the particular solution, respectively. The CSRBF [3] and MQRBF [9] are listed as follows.

CSRBF:

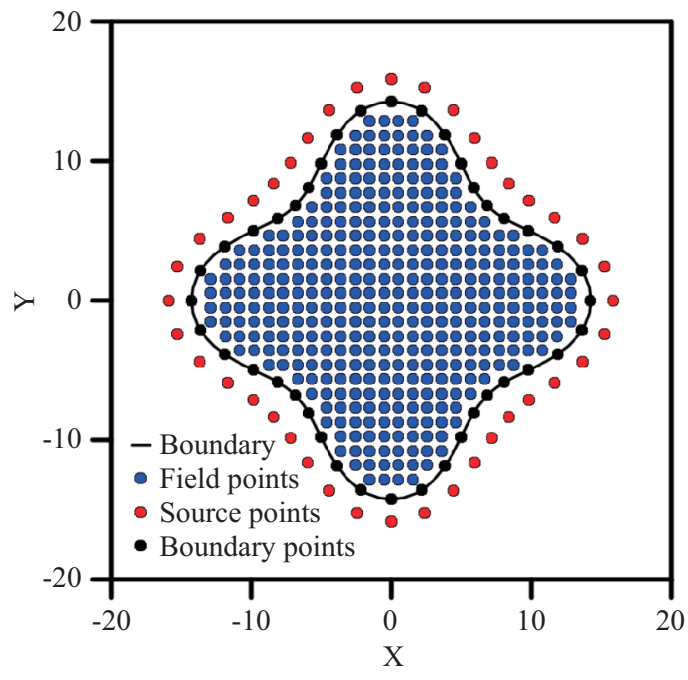

Fig. 1. Diagram of the points distribution for MPS-MFS.

$$
f(r)=\left\{\begin{array}{cc}
\left(1-\frac{r}{\lambda}\right)^{2}, & r \leq \lambda \\
0, & r>\lambda
\end{array}\right.
$$

MQRBF:

$$
f(r)=\left(r^{2}+c_{M Q}^{2}\right)^{\frac{1}{2}}
$$

where, $r$ is the distance between the field points, $\lambda$ is the scaling factor of the CSRBF, $c_{M Q}$ is the shape parameter of MQRBF. The corresponding radial basis function $F(r)$ can be obtained by analytically integrating procedure, the results of integrated RBFs are listed as follows.

Integrated CSRBF:

$F(r)=\left\{\begin{array}{l}\frac{r^{4}}{12 \lambda^{2}}-\frac{r^{3}}{3 \lambda}+\frac{r^{2}}{2}, r \leq \lambda \\ \frac{\lambda^{2}}{4}+\frac{\lambda}{3}(r-\lambda), r>\lambda\end{array}\right.$, for 1-D

$F(r)=\left\{\begin{array}{l}\frac{r^{4}}{16 \lambda^{2}}-\frac{2 r^{3}}{9 \lambda}+\frac{r^{2}}{4}, r \leq \lambda \\ \frac{13 \lambda^{2}}{144}+\frac{\lambda^{2}}{12} \ln \left(\frac{r}{\lambda}\right), r>\lambda\end{array}\right.$, for 2-D

$F(r)=\left\{\begin{array}{c}\frac{r^{4}}{20 \lambda^{2}}-\frac{r^{3}}{6 \lambda}+\frac{r^{2}}{6}, r \leq \lambda \\ \frac{\lambda^{2}}{12}-\frac{\lambda^{3}}{30 r}, r>\lambda\end{array}\right.$, for 3-D

Integrated MQRBF: 


$$
\begin{aligned}
& F(r)= \frac{1}{6}\left(r^{2}-2 c_{M Q}^{2}\right)\left(r^{2}+c_{M Q}^{2}\right)^{\frac{1}{2}} \\
&+\frac{r c_{M Q}^{2}}{2} \ln \left(r+\left(r^{2}+c_{M Q}^{2}\right)^{\frac{1}{2}}\right), \text { for 1-D } \\
& F(r)= \frac{1}{9}\left(r^{2}+4 c_{M Q}^{2}\right)\left(r^{2}+c_{M Q}^{2}\right)^{\frac{1}{2}} \\
&-\frac{c_{M Q}^{3}}{3} \ln \left(c_{M Q}+\left(r^{2}+c_{M Q}^{2}\right)^{\frac{1}{2}}\right), \text { for 2-D } \\
& F(r)=\left\{\begin{array}{l}
\frac{1}{24}\left(2 r^{2}+5 c_{M Q}^{2}\right)\left(r^{2}+c_{M Q}^{2}\right)^{\frac{1}{2}} \\
+\frac{c^{4}}{8 r} \ln \left(\frac{r+\left(r^{2}+c_{M Q}^{2}\right)^{\frac{1}{2}}}{c_{M Q}}\right), r>0, \text { for 3-D } \\
\frac{c^{3}}{3}, \quad r=0,
\end{array}\right.
\end{aligned}
$$

The homogeneous solution can be obtained by the linear combination of fundamental solutions:

$$
\phi_{H}^{n+1}=\sum_{j=1}^{N_{B}} \alpha_{j}^{n+1} G(\|\vec{x}-\vec{\xi}\|)
$$

where, $N_{B}$ is the number of the boundary points, the subscript $j$ denotes the index of the source points, $\alpha_{j}$ is the intensity of the source points and $G$ is the fundamental solution (also called the free-space Green's function) which can be written as follows:

$$
G\left(\left\|\vec{x}-\vec{\xi}_{j}\right\|\right)=\left\{\begin{array}{rr}
\frac{1}{2}\|x-\xi\|, & \text { for 1-D } \\
\frac{-1}{2 \pi} \ln (\|\vec{x}-\vec{\xi}\|), & \text { for 2-D } \\
\frac{1}{4 \pi\|\vec{x}-\vec{\xi}\|}, & \text { for 3-D }
\end{array}\right.
$$

Were $\vec{x}$ and $\vec{\xi}$ are the space-location of the field and source points, respectively. In Eq. (19), the \|\| denoted the Euclidean norm. According to the definitions of particular solutions and homogeneous solutions, we can rewrite the Eqs. (6) and (7) as follows.

$$
\begin{aligned}
\sum_{j=1}^{N_{F}} \beta_{j}^{n+1}\left(f\left(r_{j}\right)-\frac{2 F\left(r_{j}\right)}{c^{2} \Delta t^{2}}\right)_{j} & \\
& -\sum_{j=1}^{N_{B}} \alpha_{j}^{n+1} \frac{2 G\left(\left\|\vec{x}-\vec{\xi}_{j}\right\|\right)}{c^{2} \Delta t^{2}}=\frac{\left(-5 \phi^{n}+4 \phi^{n-1}-\phi^{n-2}\right)}{c^{2} \Delta t^{2}},
\end{aligned}
$$

and

$$
\left.\left[\sum_{j=1}^{N_{F}} \beta_{j}^{n+1} B\left(F\left(r_{j}\right)\right)+\sum_{j=1}^{N_{B}} \alpha_{j}^{n+1} B\left(G\left(\left\|\vec{x}-\vec{\xi}_{j}\right\|\right)\right)\right]\right|_{\vec{x} \in \partial \Omega}=b\left(\vec{x}, t^{n+1}\right) .
$$

The linear system can be written in the following matrix form:

$$
\left[\begin{array}{ll}
A_{1} & A_{2} \\
A_{3} & A_{4}
\end{array}\right]\left\{\begin{array}{l}
\beta^{n+1} \\
\alpha^{n+1}
\end{array}\right\}=\left\{\begin{array}{l}
S \\
b
\end{array}\right\}
$$

where, the sub-elements of the linear system are

$$
\begin{aligned}
& A_{1}=f+C F, A_{2}=C G, A_{3}=B(F), \\
& A_{4}=B(G), C=\frac{-2}{(c \Delta t)^{2}}, S=\frac{1}{c^{2} \Delta t^{2}}\left(-5 \phi^{n}+4 \phi^{n-1}-\phi^{n-2}\right) .
\end{aligned}
$$

In order to deal with the setup problem, the Euler scheme is used for taking the subcomponents $\phi^{n-1}$ and $\phi^{n-2}$ into the vector $S$ as follows:

$$
\left\{\begin{array}{l}
\phi^{n-1}=I_{I}-\Delta t \cdot I_{I I} \\
\phi^{n-2}=I_{I}-2 \Delta t \cdot I_{I I}
\end{array}, n \leq 2\right.
$$

The sub-vectors $\beta$ and $\alpha$ can be obtained by solving the linear system as the Eq. (22). After the linear system is solved, the solution in the computational domain can be obtained from the definitions of particular solutions and homogeneous solutions as the following:

$$
\phi^{n+1}=\sum_{j=1}^{N_{F}} \beta_{j}^{n+1} F\left(r_{j}\right)+\sum_{j=1}^{N_{B}} \alpha_{j}^{n+1} G\left(\left\|\vec{x}-\vec{\xi}_{j}\right\|\right) .
$$

\section{NUMERICAL EXAMPLE}

The proposed meshless method is tested by considering three numerical examples. These are analyzed and validated to prove the accuracy and efficiency of the proposed meshless numerical scheme and also to display the advantages of the proposed wave model. We use the $L^{2}$ norm error $\left(E_{L}^{2}\right)$ to estimate the accuracy, which is defined as:

$$
E_{L^{2}}=\left(\frac{\sum_{i=1}^{N_{T}}\left(\phi_{i, \text { Analytical }}-\phi_{i, \text { Numerical }}\right)^{2}}{\sum_{i=1}^{N_{T}} \phi_{i, \text { Analytical }}^{2}}\right)^{\frac{1}{2}},
$$

where $N_{T}$ is the number of the resolve points. 
Table 1. The $E_{L^{2}}$ comparisons for the 1D wave problem.

\begin{tabular}{|c|c|c|c|c|c|c|c|c|}
\hline \multirow{3}{*}{$\frac{\text { Model }}{\Delta t}$} & \multirow{2}{*}{\multicolumn{2}{|c|}{ FDM }} & \multirow{2}{*}{\multicolumn{2}{|c|}{ ELMFS }} & \multicolumn{4}{|c|}{ MPS-MFS } \\
\hline & & & & & \multicolumn{2}{|c|}{ CSRBF } & \multicolumn{2}{|c|}{ MQRBF } \\
\hline & 0.05 & 0.02 & 0.05 & 0.02 & 0.05 & 0.02 & 0.05 & 0.02 \\
\hline$t=2.5$ & $9.18 \mathrm{E}-02$ & $3.39 \mathrm{E}-02$ & $6.24 \mathrm{E}-03$ & $4.56 \mathrm{E}-03$ & $2.13 \mathrm{E}-02$ & $6.53 \mathrm{E}-04$ & $2.15 \mathrm{E}-02$ & $5.01 \mathrm{E}-04$ \\
\hline$t=5$ & $5.72 \mathrm{E}-02$ & $2.46 \mathrm{E}-02$ & 7.43E-03 & $4.16 \mathrm{E}-03$ & $1.79 \mathrm{E}-02$ & $1.02 \mathrm{E}-03$ & $1.82 \mathrm{E}-02$ & $7.95 \mathrm{E}-04$ \\
\hline$t=7.5$ & $1.27 \mathrm{E}-01$ & $4.13 \mathrm{E}-02$ & $1.32 \mathrm{E}-02$ & $8.15 \mathrm{E}-03$ & $5.07 \mathrm{E}-02$ & $8.75 \mathrm{E}-04$ & $5.12 \mathrm{E}-02$ & $4.82 \mathrm{E}-04$ \\
\hline$t=10$ & $1.40 \mathrm{E}-01$ & $5.27 \mathrm{E}-02$ & $1.16 \mathrm{E}-02$ & $9.96 \mathrm{E}-03$ & $6.56 \mathrm{E}-02$ & $2.20 \mathrm{E}-03$ & $6.63 \mathrm{E}-02$ & $1.43 \mathrm{E}-03$ \\
\hline
\end{tabular}

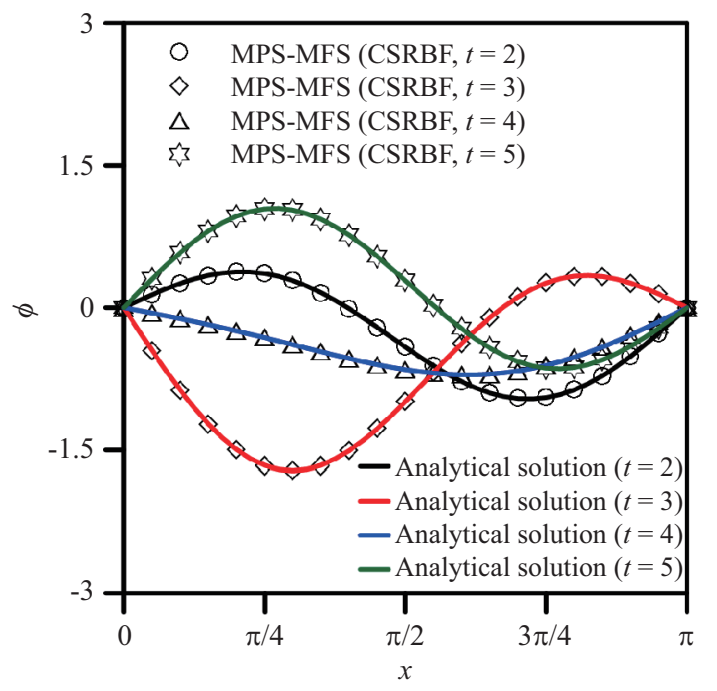

Fig. 2. The evolution of displacement for the string vibration problem by CSRBF.

\section{String Vibration Problem}

In the first example, the string vibration problem is selected to test the performances of the wave models in one-dimensional domain. In this example, the wave speed $c=1$ is selected in wave field for simulation. The computational domain is $0<x<$ $\pi$. The initial conditions are selected as:

$$
\left.\phi(x, t)\right|_{t=0}=\sin x-\sin 2 x \text { and }\left.\frac{\partial \phi(x, t)}{\partial t}\right|_{t=0}=0 .
$$

The fixed boundary conditions are chosen as the following forms:

$$
\left.\phi(x, t)\right|_{x=0}=0 \text { and }\left.\phi(x, t)\right|_{x=\pi}=0 .
$$

The analytical solution is obtained by the method of separation of variables:

$$
\phi(x, t)=\cos t \sin x-\cos 2 t \sin 2 x .
$$

In this case, the ELMFS, the MPS-MFS and the FDM wave models used 41 points for calculation and the time-interval $\Delta t$

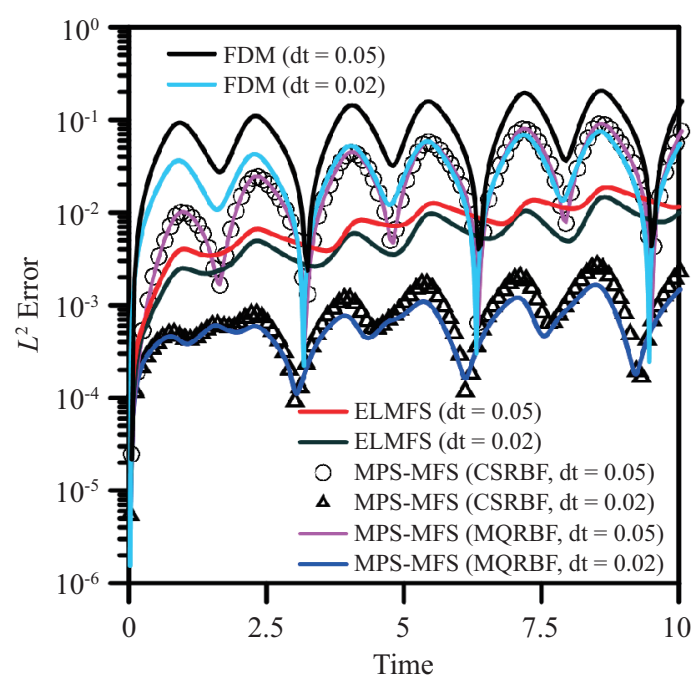

Fig. 3. The $E_{L}^{2}$ of the string vibration problem.

equal to 0.05 and 0.02 are selected for comparison. Fig. 2 displays the numerical results by the MPS-MFS wave model with CSRBF and time-interval $\Delta t=0.02$. Fig. 3 depicted the history of $E_{L}^{2}$ by the ELMFS, the MPS-MFS and the FDM wave models. From the Table 1 and Fig. 3, it is clearly shown that the error curves of the FDM model always oscillate near $10^{-2}$. And also, we detect that the accuracy of ELMFS and MPS-MFS models are better than FDM model. At the same time, the effects of the time-interval for ELMFS model are not very sensitive. When the time-interval $\Delta t=0.05$ is used for MPS-MFS model, the error curves almost cover with the error curve of FDM $(\Delta t=0.02)$. The best result is obtained by MPS-MFS model with time-interval $\Delta t=0.02$. From this numerical case, we consider the performance of ELMFS and MPS-MFS models are better than the FDM model. Besides, the accuracy of MQRBF for MPS-MFS model is better than CSRBF (but not much difference) in this case. It is convinced that the proposed MPS-MFS model is a high accuracy wave model.

\section{Wave Vibration in 2-D Irregular Domain}

We next consider a more complicated wave vibrating problem in an irregular domain with a smooth edge and wave speed $c=1$. The computational domain $\Omega$ and its boundary $\partial \Omega$ are defined as follows: 
Table 2. The $E_{L}{ }^{2}$ comparisons for the 2D wave problem.

\begin{tabular}{|c|c|c|c|c|c|c|c|}
\hline \multirow{3}{*}{$\begin{array}{l}\text { Model } \\
\text { Points }\end{array}$} & \multirow{2}{*}{\multicolumn{3}{|c|}{ FEM }} & \multicolumn{4}{|c|}{ MPS-MFS } \\
\hline & & & & \multicolumn{2}{|c|}{ CSRBF } & \multicolumn{2}{|c|}{ MQRBF } \\
\hline & 4,693 & 11,339 & 15,981 & 433 & 1,209 & 433 & 1,209 \\
\hline$t=12$ & $6.24 \mathrm{E}-04$ & $2.44 \mathrm{E}-04$ & $1.18 \mathrm{E}-04$ & $1.03 \mathrm{E}-03$ & $2.21 \mathrm{E}-04$ & $1.11 \mathrm{E}-03$ & $2.81 \mathrm{E}-04$ \\
\hline$t=24$ & $2.23 \mathrm{E}-04$ & $8.52 \mathrm{E}-05$ & $3.70 \mathrm{E}-05$ & $1.10 \mathrm{E}-03$ & $2.10 \mathrm{E}-04$ & $1.18 \mathrm{E}-03$ & $2.48 \mathrm{E}-04$ \\
\hline$t=36$ & $1.53 \mathrm{E}-03$ & $5.87 \mathrm{E}-04$ & $2.67 \mathrm{E}-04$ & $8.50 \mathrm{E}-04$ & $2.68 \mathrm{E}-04$ & 8.92E-04 & $3.97 \mathrm{E}-04$ \\
\hline$t=48$ & $2.94 \mathrm{E}-03$ & $1.13 \mathrm{E}-03$ & $5.27 \mathrm{E}-04$ & $1.40 \mathrm{E}-03$ & 4.44E-04 & $1.48 \mathrm{E}-03$ & $6.98 \mathrm{E}-04$ \\
\hline$t=60$ & $2.24 \mathrm{E}-03$ & $8.59 \mathrm{E}-04$ & $4.07 \mathrm{E}-04$ & $1.27 \mathrm{E}-03$ & $3.75 \mathrm{E}-04$ & $1.38 \mathrm{E}-03$ & $5.90 \mathrm{E}-04$ \\
\hline
\end{tabular}

$$
\partial \Omega=\left\{(x, y) \mid \begin{array}{l}
x=R_{C} \cos \theta \\
y=R_{C} \sin \theta
\end{array}, 0 \leq \theta<2 \pi\right\}
$$

where, $R_{C}$ is defined by:

$$
R_{C}=10\left[\cos 4 \theta+\left(\frac{18}{5}-\sin ^{2} 4 \theta\right)^{\frac{1}{2}}\right]^{\frac{1}{3}}
$$

The initial displacement and velocity are selected as follows:

$$
\left.\phi(x, y, t)\right|_{t=0}=3 \text { and }\left.\frac{\partial \phi(x, y, t)}{\partial t}\right|_{t=0}=\frac{\sqrt{2} \pi}{10} \cos \frac{\pi x}{10} \cos \frac{\pi y}{10}
$$

The boundary condition is given as:

$$
\left.\phi(x, y, t)\right|_{(x, y) \in \partial \Omega}=3+\cos \frac{\pi x}{10} \cos \frac{\pi y}{10} \sin \frac{\sqrt{2} \pi t}{10},
$$

and the analytical solution is:

$$
\phi(x, y, t)=3+\cos \frac{\pi x}{10} \cos \frac{\pi y}{10} \sin \frac{\sqrt{2} \pi t}{10} .
$$

In this case, we used 433 and 1,209 points for MPS-MFS model calculation. In order to verify the correctness of numerical results for the proposed MPS-MFS wave model, we also solved the same problem by the FEM (combine with the Houbolt FD scheme and linear triangle elements with 4,693, 11,339 and 15,981 points). Fig. 4 depicts the evolution of the $E_{L}^{2}$ obtained by the MPS-MFS and the FEM. We discover that there is no large difference between the error curves from the MPS-MFS (by CSRBF with 1,209 points) and the FEM $(15,981$ points). Besides, the accuracy of MPS-MFS by CSRBF is better than MQRBF in this case (but not very large). In the $E_{L}^{2}$ curve obtained by the MPS-MFS model (433 points), the error curves always oscillate near $10^{-3}$. Besides, the MPS-MPS model shows the good performance with smaller number of points $(1,209$ points) than the FEM results with larger number

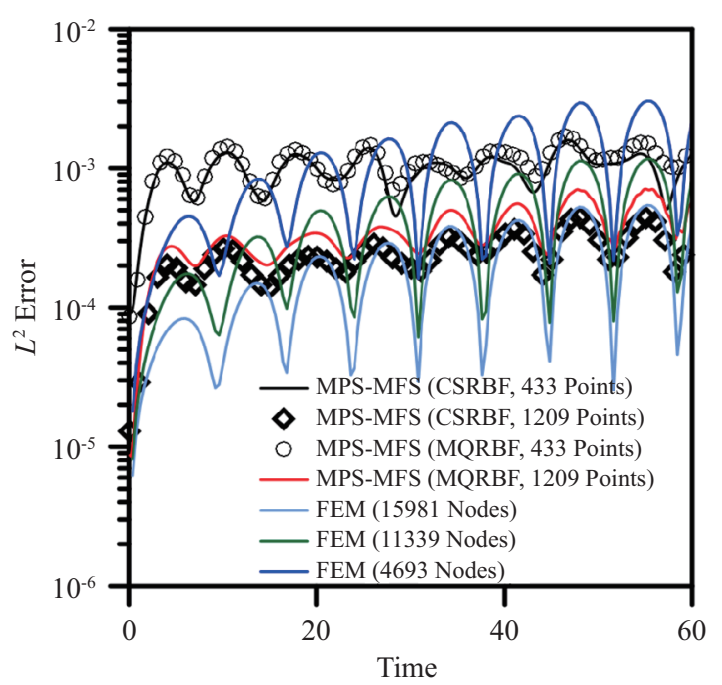

Fig. 4. The $E_{L}^{2}$ history of the two-dimensional wave vibration problem.

of points $(15,981$ points) as shown in Table 2 . In this test, the proposed time-marching MPS-MFS model uses very few collocation points to deal with this irregular-domain problem and still yields as accurate results as the FEM, which uses a very dense mesh and many points.

Furthermore, we change the wave speed $(c=1.5)$, initial and boundary conditions to test the wave propagation problem with the same domain shape. The hump shape of the initial displacement and zero initial velocity are listed as follows:

$$
\left.\phi(x, y, t)\right|_{t=0}=e^{\frac{-4}{25}\left[(x+5)^{2}+y^{2}\right]} \text { and }\left.\frac{\partial \phi(x, y, t)}{\partial t}\right|_{t=0}=0,
$$

and the fixed boundary condition is:

$$
\left.\phi(x, y, t)\right|_{(x, y) \in \partial \Omega}=0 .
$$

We used 1,209 collocation points with a time-interval $\Delta t=$ $8 \times 10^{-3}$ to simulate this problem. The same problem was also simulated by the FEM with mesh dependent results $(11,339$ and 15,981 points). Figs. 5 (a)-(d) displayed the evolution history of the displacement at $(x, y)=(0,5),(0,13),(0,0)$ and $(-5,-5)$, respectively. From Figs. 5 (a)-(d), we can observe 

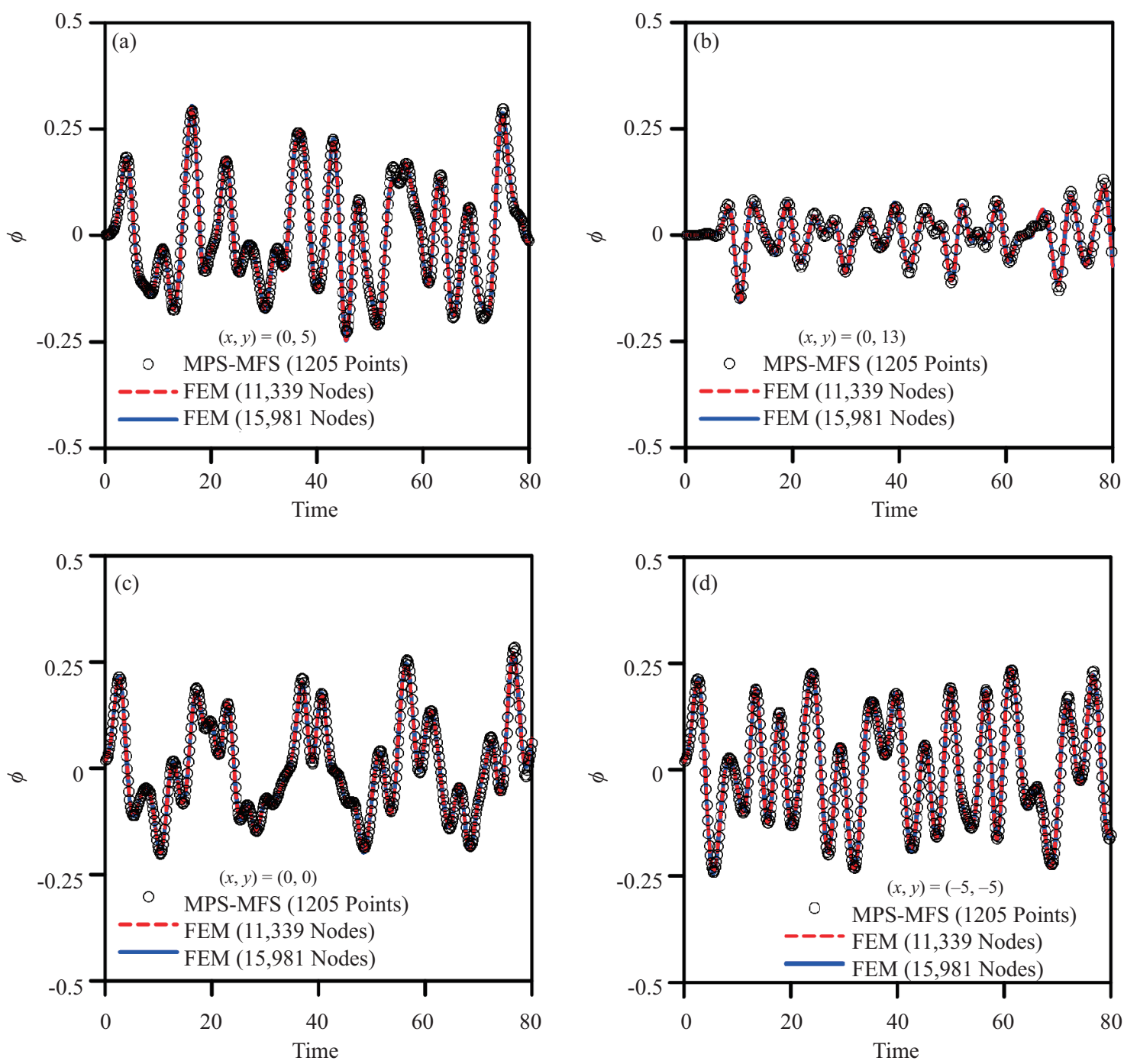

Fig. 5. The potential time history of the wave propagation problem (a) at $(0,5),(b)$ at $(0,13)$, (c) at $(0,0)$, and $(d)$ at $(-5,-5)$.

that the solutions from MPS-MFS are still similar to the results by FEM, however the present method only use very small amount of points and one order of points is used by the FEM. Figs. 6 (a)-(f) depict the evolution of the complex wave phenomena.

\section{Wave Vibration in 3-D Irregular Domain}

We next consider a wave vibration problem in a threedimensional irregular domain without boundary effects. In this simulation, the wave speed $c=2$ is selected. The computational domain boundary is described as the following:

$$
\partial \Omega=\left\{\begin{aligned}
x & =R_{C} \cos \theta \\
(x, y, z) \mid y & =R_{C} \sin \theta \sin \varphi, 0 \leq \theta<2 \pi, 0 \leq \varphi<\pi \\
z & =R_{C} \sin \theta \cos \varphi
\end{aligned}\right\},
$$

where, $R_{C}$ is defined as:

$$
R_{C}=5\left[\cos 3 \theta+\left(8-\sin ^{2} 3 \theta\right)^{\frac{1}{2}}\right]^{\frac{1}{3}}
$$

The initial potential and initial velocity are selected as:

$$
\begin{aligned}
& \left.\phi(x, y, z, t)\right|_{t=0}=0 \\
& \left.\frac{\partial \phi(x, y, z, t)}{\partial t}\right|_{t=0}=\frac{\sqrt{3} \pi}{4} \cos \frac{\pi x}{8} \cos \frac{\pi y}{8} \cos \frac{\pi z}{8},
\end{aligned}
$$

and the boundary condition is obtained from the analytical solution which is:

$$
\phi(x, y, z, t)=\cos \frac{\pi x}{8} \cos \frac{\pi y}{8} \cos \frac{\pi z}{8} \sin \frac{\sqrt{3} \pi t}{4}
$$




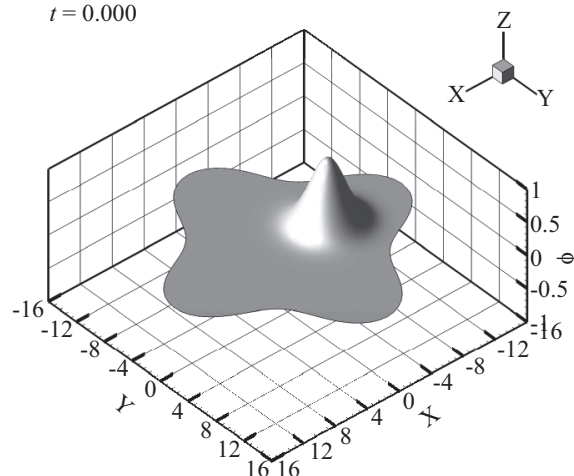

(a)

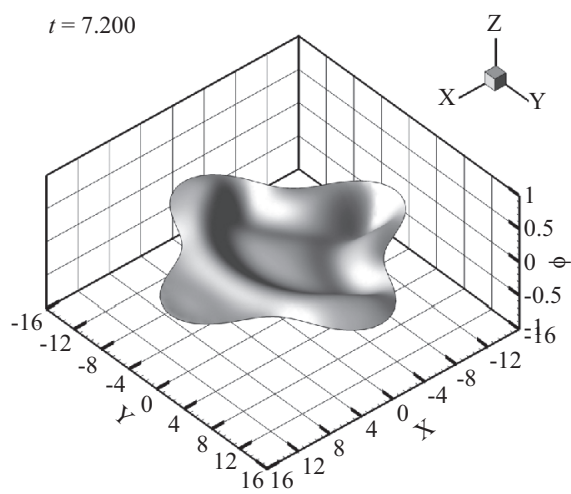

(d)

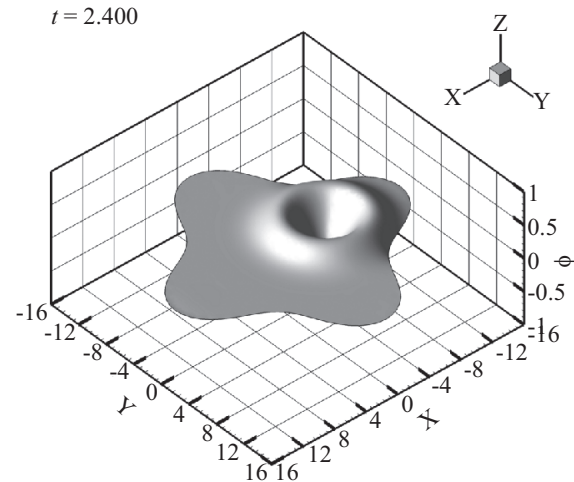

(b)

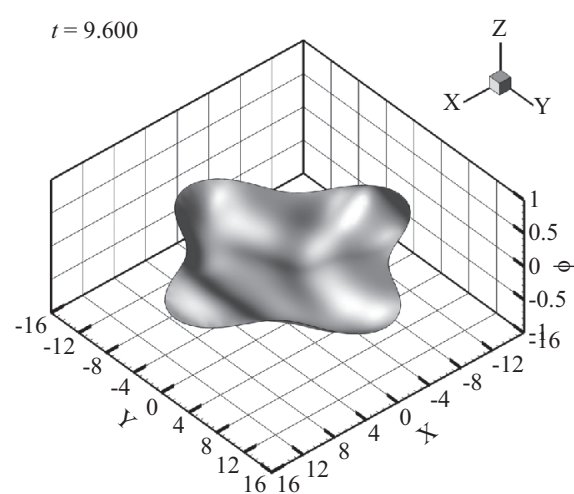

(e)

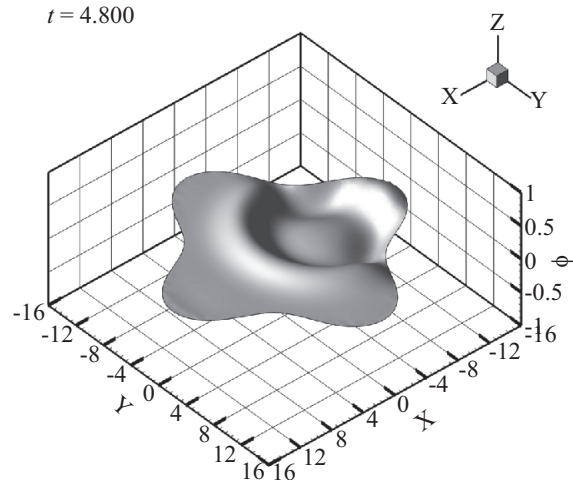

(c)

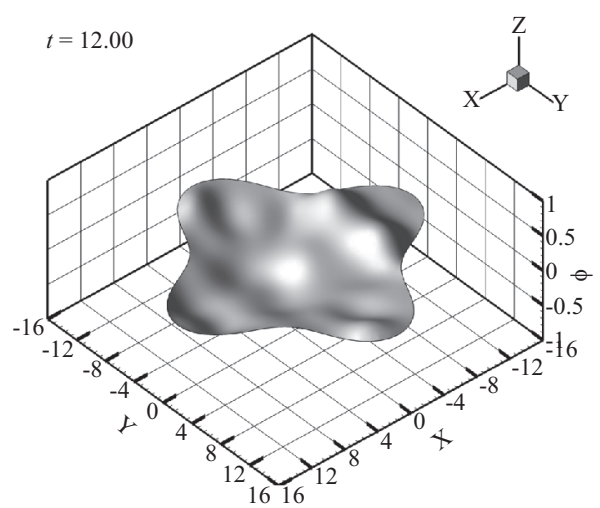

(f)

Fig. 6. The evolution of the wave propagation problem (a) $t=0$, (b) $t=2.4$, (c) $t=4.8$, (d) $t=7.2$, (e) $t=9.6$, and (f) $t=12$.

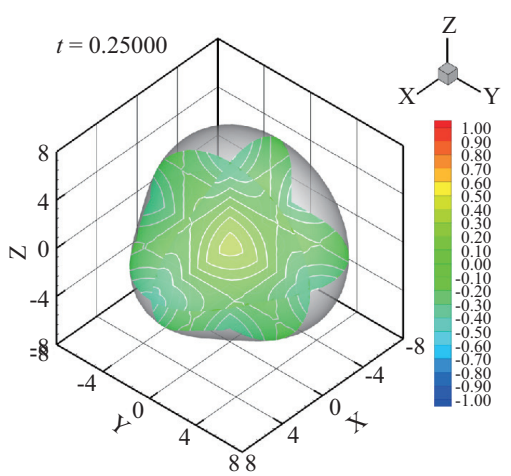

(a)

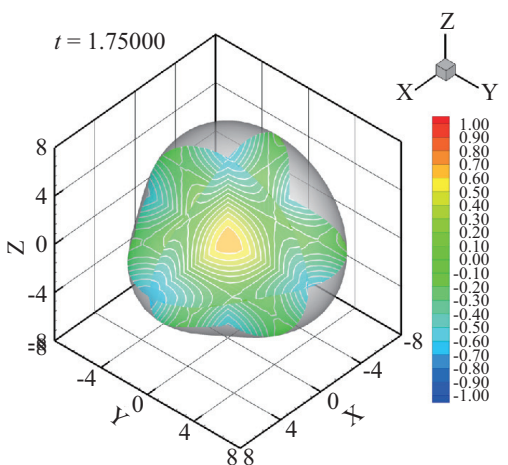

(d)

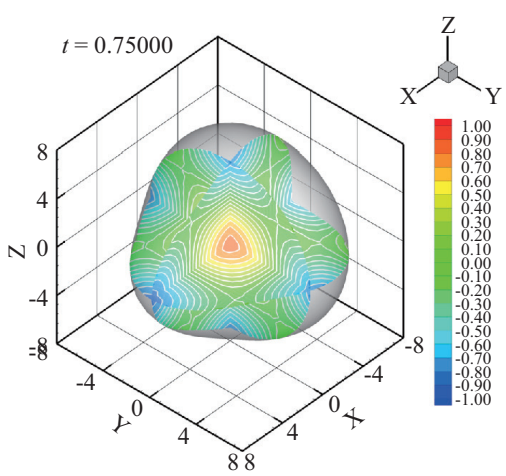

(b)

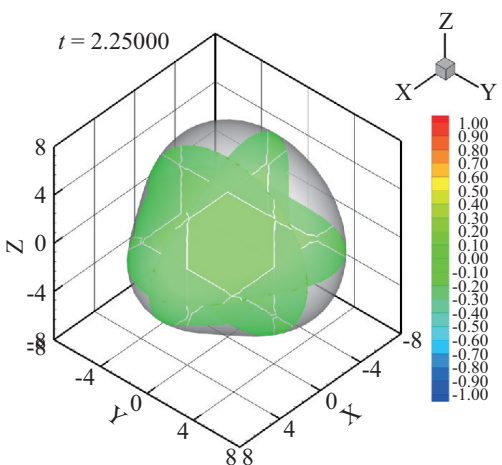

(e)

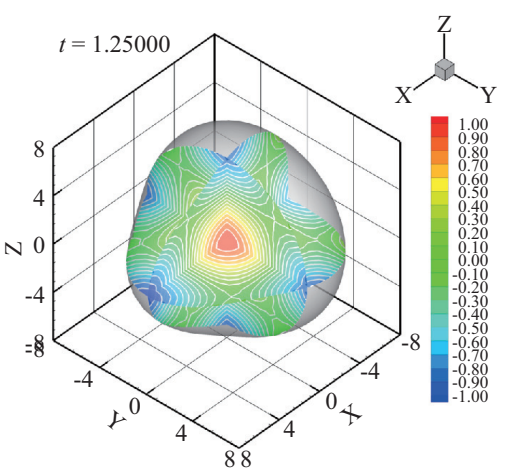

(c)

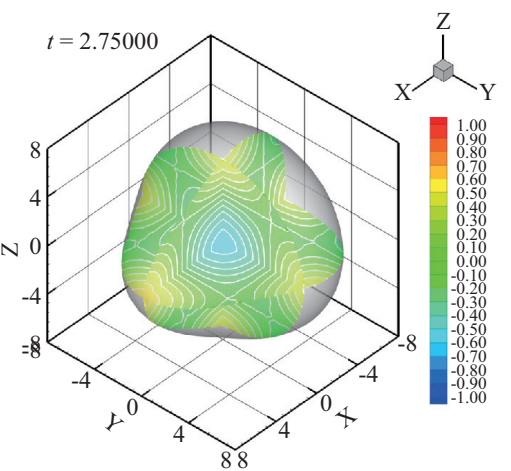

(f)

Fig. 7. The evolution of the wave potential in 3D irregular problem (a) $t=0.25$, (b) $t=0.75$, (c) $t=1.25$, (d) $t=1.75$, (e) $t=2.25$, and (f) $t=2.75$. 
Table 3. The $E_{L^{2}}$ comparisons for the $3 \mathrm{D}$ wave problem.

\begin{tabular}{|c|c|c|c|c|c|c|c|}
\hline \multirow{3}{*}{$\begin{array}{l}\text { Model } \\
\text { Points }\end{array}$} & \multirow{2}{*}{\multicolumn{3}{|c|}{ FEM }} & \multicolumn{4}{|c|}{ MPS-MFS } \\
\hline & & & & \multicolumn{2}{|c|}{ CSRBF } & \multicolumn{2}{|c|}{ MQRBF } \\
\hline & 10,786 & 21,783 & 32,526 & 1,389 & 3,233 & 1,389 & 3,233 \\
\hline$t=5$ & $3.70 \mathrm{E}-03$ & $2.30 \mathrm{E}-03$ & $1.73 \mathrm{E}-03$ & $1.14 \mathrm{E}-03$ & 1.19E-04 & 8.83E-04 & $9.48 \mathrm{E}-05$ \\
\hline$t=10$ & $4.38 \mathrm{E}-03$ & $2.64 \mathrm{E}-03$ & $1.96 \mathrm{E}-03$ & $1.90 \mathrm{E}-03$ & $2.40 \mathrm{E}-04$ & $1.44 \mathrm{E}-03$ & $2.18 \mathrm{E}-04$ \\
\hline$t=15$ & $2.42 \mathrm{E}-03$ & $1.30 \mathrm{E}-03$ & 8.84E-04 & $1.44 \mathrm{E}-03$ & $1.68 \mathrm{E}-04$ & $1.19 \mathrm{E}-03$ & $1.40 \mathrm{E}-04$ \\
\hline$t=20$ & $3.88 \mathrm{E}-03$ & $2.72 \mathrm{E}-03$ & $2.08 \mathrm{E}-03$ & $1.16 \mathrm{E}-03$ & $1.73 \mathrm{E}-04$ & 7.95E-04 & $1.71 \mathrm{E}-04$ \\
\hline$t=25$ & $1.12 \mathrm{E}-02$ & $7.30 \mathrm{E}-03$ & $5.56 \mathrm{E}-03$ & $2.23 \mathrm{E}-03$ & $2.30 \mathrm{E}-04$ & $1.65 \mathrm{E}-03$ & $1.63 \mathrm{E}-04$ \\
\hline
\end{tabular}

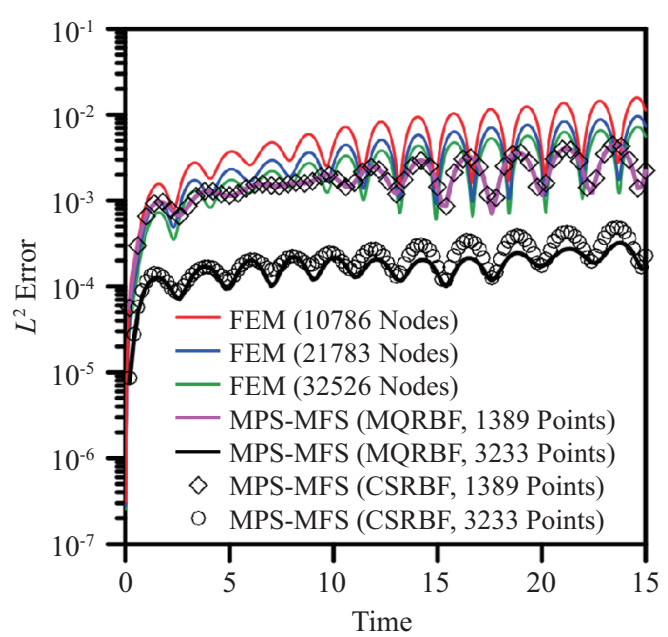

Fig. 8. The $E_{L}{ }^{2}$ of the three-dimensional wave problem.

In this case, we used 1,389 collocation points (with 361 boundary points), as well as 3,233 collocation points (with 648 boundary points) and a time-interval $\Delta t=2.5 \times 10^{-3}$. In order to demonstrate the accuracy of numerical results, the same problem is solved by the FEM with linear tetrahedral by 10,786 points, 21,783 points and 32,526 points, respectively. In Figs. 7 (a)-(f), we present the MPS-MFS solution profiles at $x=0, y=0$ and $z=0$ which are obtained by MQRBF. Fig. 8 depicts the evolution of the $E_{L}{ }^{2}$ obtained by the MPS-MFS and the FEM. From Fig. 8 and Table $3, E_{L^{2}}$ by the MPS-MFS $(1,389$ points $)$ and the FEM (32,526 points) are almost the same. In the $E_{L}{ }^{2}$ curve obtained with the MPS-MFS (both CSRBF and MQRBF, 3,233 points), the error curves always oscillate near $10^{-4}$ and are smaller than the FEM results with a larger number of points. And also, the accuracy of MPS-MFS model by MQRBF is better than CSRBF in this case (but not very large). From these comparisons, we conclude that the proposed MPS-MFS model is a highly accurate and efficient numerical tool for solving the multi-dimensional wave problem with irregular domain even when using very few collocation points.

\section{CONCLUSION}

In this paper, a novel numerical method based on the MPS, MFS and Houbolt FD method is developed to approximate the solutions of multi-dimensional wave equations. The Houbolt method is used to avoid the difficulty of constructing the linear algebraic system of the Cauchy conditions. The resulting partial differential equations are handled by the coupled MPSMFS model. In addition, the proposed meshless model is free from the numerical quadrature and mesh generation. Three numerical examples in one-, two- and three-dimensional domains are selected to verify the efficacy of the proposed method. From the numerical results, it can be observed that accurate solutions in irregular domains can be obtained easily by the proposed method with very few collocation points. The numerical results demonstrate the accuracy, consistency and applicability of the proposed meshless numerical model for multi-dimensional wave equations with irregular geometries.

\section{ACKNOWLEDGMENTS}

The National Science Council (NSC) of Taiwan is gratefully acknowledged for providing the financial support to carry out the present work under the grant number: NSC-972221-E-002-247-MY3 and NSC-96-2221-E-002-127-MY3. We also deeply appreciated about some importance suggestions by Professor C.S. Chen in Department of Mathematics University of Southern Mississippi, USA.

\section{REFERENCES}

1. Alves, C. J. S. and Chen, C. S., "A new method of fundamental solutions applied to nonhomogeneous elliptic problems," Advances in Computational Mathematics, Vol. 23, No. 1-2, pp. 125-142 (2005).

2. Chen, C. S., "The method of fundamental solutions for nonlinear thermal explosion," Communications in Numerical Methods in Engineering, Vol. 11, No. 8, pp. 675-681 (1995).

3. Chen, C. S., Brebbia, C. A., and Power, H., "Dual reciprocity method using compactly supported radial basis functions," Communications in Numerical Method in Engineering, Vol. 15, No. 2, pp. 137-150 (1999).

4. Chen, J. T., Chou, K. S., and Kao, S. K., "One-dimensional wave animation using Mathematica," Computer Applications in Engineering Education, Vol. 17, No. 3, pp. 323-339 (2009).

5. Chen, J. T., Lee, Y. T., Yu, S. R., and Shieh, S. C., "Equivalence between Trefftz method and method of fundamental solution for the annular Green's function using the addition theorem and image concept," Engineering Analysis with Boundary Elements, Vol. 33, No. 5, pp. 678-688 (2009).

6. Chen, J. T., Wu, C. S., Lee, Y. T., and Chen, K. H., "On the equivalence of the Trefftz method and method of fundamental solutions for Laplace and biharmonic equations," Computers and Mathematics with Applications, Vol. 53, No. 6, pp. 851-879 (2007). 
7. Chen, K. H., Chen, J. T., and Kao, J. H., "Regularized meshless method for antiplane shear problems," International Journal for Numerical Methods in Engineering, Vol. 73, No. 9, pp. 1251-1273 (2008).

8. Chen, Y. W., Liu, C. S., and Chang, J. R., "Applications of the modified Trefftz method for the Laplace equation," Engineering Analysis with Boundary Elements, Vol. 33, No. 2, pp. 137-146 (2009).

9. Cheng, A. H. D., Golberg, M. A., Kansa, E. J., and Zammito, G., "Exponential convergence and H-c multiquadric collocation method for partial differential equations," Numerical Methods for Partial Differential Equations, Vol. 19, No. 5, pp. 571-594 (2003).

10. Cho, H. A., Golberg, M. A., Muleshkov, A. S., and Li, X., "Trefftz methods for time dependent partial differential equations," CMC-Computers Materials \& Continua, Vol. 1, No. 1, pp. 1-37 (2004).

11. Golberg, M. A., "The method of fundamental solutions for Poisson's equations," Engineering Analysis with Boundary Elements, Vol. 16, No. 3 pp. 205-213 (1995)

12. Gu, M. H., Young, D. L., and Fan, C. M., "The meshless method for onedimensional hyperbolic equation," Journal of Aeronautics, Astronautics and Aviation, Series A, Vol. 40, No. 2, pp. 63-72 (2008).

13. Gu, M. H., Young, D. L., and Fan, C. M., "The method of fundamental solutions for one-dimensional wave equations," CMC-Computers Materials \& Continua, Vol. 11, No. 3, pp. 185-208 (2009).

14. Houbolt, J. C., "A recurrence matrix solution for the dynamic response of elastic aircraft," Journal of the Aeronautical Sciences, Vol. 17, pp. 540550 (1950).

15. Jiang, F., Oliveira, M. S. A., and Sousa, A. C. M., "Mesoscale SPH modeling of fluid flow in isotropic porous media," Computer Physics Communications, Vol. 176, No. 7, pp. 471-480 (2007).

16. Kim, H. C. and Verboncoeur, J. P., "Reflection, absorption and transmission of TE electromagnetic waves propagation in a nonuniform plasma slab,” Computer Physics Communications, Vol. 177, No. 1-2, pp. 118-121 (2007).

17. Kupradze, V. D. and Aleksidze, M. A., "The method of functional equations for the approximate solution of certain boundary value problem," USSR Computational Mathematics and Mathematical Physics, Vol. 4 , No. 4, pp. 82-126 (1964).

18. Mathon, R. and Johnston, R. L., "The approximate solution of elliptic boundary-value problems by fundamental solutions," SIAM Journal on Numerical Analysis, Vol. 14, No. 4, pp. 638-650 (1977).

19. Popovich, P., Cooper, W. A., and Villard, L., "A full-wave solver of the Maxwell's equations in 3D cold plasmas," Computer Physics Communications, Vol. 175, No. 4, pp. 250-263 (2006).

20. Soroushian, A. and Farjoodi, J., "A unified starting procedure for the
Houbolt method," Communications in Numerical Methods in Engineering, Vol. 24, No. 1, pp. 1-13 (2008).

21. Tanaka, M. and Chen, W., "Dual reciprocity BEM applied to transient elastodynamic problems with differential quadrature method in time," Computer Methods in Applied Mechanics and Engineering, Vol. 190, No. 18-19, pp. 2331-2347 (2001).

22. Valtchev, S. S. and Roberty, N. C., "A time-marching MFS scheme for heat conduction problems," Engineering Analysis with Boundary Elements, Vol. 32, No. 6, pp. 480-493 (2008).

23. Wang, H., Qin, Q. H., and Kang, Y. L., "A meshless model for transient heat conduction in functionally graded materials," Computational $\mathrm{Me}$ chanics, Vol. 38, No. 1, pp. 51-60 (2006).

24. Wazwaz, A. M., "A reliable technique for solving the wave equation in an infinite one-dimensional medium," Applied Mathematics and Computation, Vol. 92, No. 1, pp. 1-7 (1998).

25. Wu, T. Y., A Study on Time-Discontinuous Galerkin Finite Element Method for Elastodynamic Problems, Ph.D. Dissertation, Chung Yuan Christian University, Chung Li, Taiwan (2001).

26. Young, D. L., Chen, C. H., Fan, C. M., and Shen, L. H., "The method of fundamental solutions with eigenfunctions expansion method for $3 \mathrm{D}$ nonhomogeneous diffusion equations," Numerical Methods for Partial Differential Equations, Vol. 25, No. 1, pp. 195-211 (2009).

27. Young, D. L., Chen, K. H., and Lee, C. W., "Novel meshless method for solving the potential problems with arbitrary domain," Journal of Computational Physics, Vol. 209, No. 1, pp. 290-321 (2005).

28. Young, D. L., Fan, C. M., Hu, S. P., and Atluri, S. N., "The EulerianLagrangian method of fundamental solutions for two-dimensional unsteady Burgers' equations," Engineering Analysis with Boundary Elements, Vol. 32, No. 5, pp. 395-412 (2008).

29. Young, D. L., Fan, C. M., Tsai, C. C., Chen, C. W., and Murugesan, K., "Eulerian-Lagrangian method of fundamental solutions for multidimensional advection-diffusion equation," International Mathematical Forum, Vol. 1, No. 13-16, pp. 687-706 (2006).

30. Young, D. L., Gu, M. H., and Fan, C. M., "The time-marching method of fundamental solutions for wave equations," Engineering Analysis with Boundary Elements, Vol. 33, No. 12, pp. 1411-1425 (2008).

31. Young, D. L., Jane, S. J., Fan, C. M., Murugesan, K., and Tsai, C. C., "The method of fundamental solutions for 2D and 3D Stokes problems," Journal of Computational Physics, Vol. 211, No. 1, pp. 1-8 (2006).

32. Young, D. L., Tsai, C. C., Murugesan, K., Fan, C. M., and Chen, C. W., "Time dependent fundamental solutions for homogeneous diffusion problems," Engineering Analysis with Boundary Elements, Vol. 28, No. 12, pp. 1463-1473 (2004). 